\title{
A Phase 2 study of cisplatin analog CI-973 in the treatment of patients with refractory, advanced ovarian cancer
}

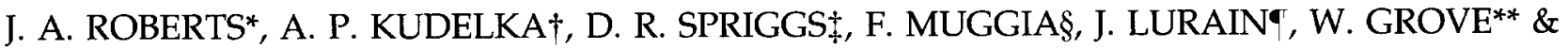 \\ C. KOWAL* \\ *Division of Gynecologic Oncology, University of Michigan, Ann Arbor, Michigan, †Section of Gynecologic Medical \\ Oncology, M.D. Anderson Cancer Center, Houston, Texas, $\ddagger$ Department of Human Oncology, University of Wisconsin, \\ Madison, Wisconsin, §Kenneth Norris Comprehensive Cancer Center, University of Southern California, Los Angeles, \\ - Section of Gynecologic Oncology, Northwestern University Medical School, Chicago, Illinois and **Parke-Davis \\ Pharmaceutical Research, Division of Warner-Lambert Company, Ann Arbor, Michigan, USA
}

\begin{abstract}
Roberts JA, Kudelka AP, Spriggs DR, Muggia F, Lurain J, Grove W, Kowal C. A Phase 2 study of cisplatin analog CI-973 in the treatment of patients with refractory, advanced ovarian cancer. Int J Gynecol Cancer 1996; 6: $257-260$.

The platinum analog CI-973 was selected for clinical study because it is associated with fewer and milder toxicities than either cisplatin or carboplatin in experimental animals, and because it has activity against cisplatin-resistant cell lines in vitro. In this Phase 2 study, 31 women with platinum-resistant or recurrent ovarian carcinoma were treated with CI-973. Three patients achieved a complete response, defined as the disappearance of all measurable disease. The median Kaplan-Meier survival time was estimated to be approximately 7 months from the start of treatment. The most frequently occurring drug-related toxicities were neutropenia, nausea, vomiting, asthenia, anemia, abdominal pain, and diarrhea. Most toxicities were mild or moderate and none resulted in withdrawal from treatment. Despite this favorable toxicity profile, the low response rate of $10 \%$ does not support further clinical development of CI-973 as treatment for ovarian cancer.
\end{abstract}

KEYWORDS: CI-973, ovarian cancer, Phase 2 study, platinum analog.

Ovarian cancer is the leading cause of gynecologic cancer deaths and the fourth leading cause of all cancer deaths among women in the USA ${ }^{(1,2)}$. Because ovarian cancer is usually asymptomatic in its early stages, most patients have widespread disease at the time of diagnosis. The yearly mortality rate is currently $65 \%$ of the incidence rate, and the 5-year survival rate in patients with stage III or IV disease is less than $10 \%$, despite aggressive surgery and chemotherapy. More than half ovarian cancer cases occur in women over 65 years of age; therefore, the incidence of this disease will likely rise as the median age of the population increases.

Address for correspondence: Dr J. A. Roberts, Division of Gynecologic Oncology, D2246 Medical Professional Building, 1500 East Medical Center Drive, Ann Arbor, Michigan, 48109-0718, USA.
Platinum analogs are one of the most widely used chemotherapeutic agents for the frontline treatment of ovarian cancer ${ }^{(3)}$. However, their effectiveness is often limited in recurrent disease because tumor cells develop resistance to further treatment. Cisplatin is associated with dose-limiting nephrotoxicity, neurotoxicity, ototoxicity, and emetogenesis, making it difficult for many patients to tolerate adequate therapy ${ }^{(4)}$. Carboplatin, which is the only other platinum-containing compound approved for clinical use, is somewhat less toxic than cisplatin, but exhibits nearly complete cross-resistance with cisplatin both in vitro and clinically.

CI-973 (NK121) is a third-generation, water-soluble cisplatin analog with antitumor activity superior or equivalent to cisplatin and carboplatin against cisplatin-resistant murine and human tumor cell lines 
in vitro and in vivo ${ }^{(4-6)}$. Phase 1 studies in patients with solid tumors indicate that, unlike cisplatin, CI-973 does not appear to cause serious renal toxicity, neurotoxicity, or ototoxicity ${ }^{(7-9)}$. CI-973 is myelosuppressive, causing dose-limiting neutropenia, but is not associated with the dose-limiting thrombocytopenia characteristic of carboplatin therapy. This multicenter Phase 2 study was performed to determine the clinical efficacy of CI-973 in patients with advanced ovarian carcinoma that has progressed or recurred after treatment with cisplatin or carboplatin, and to further define the safety profile of this experimental drug.

\section{Patients and methods}

\section{Patients}

Thirty-one patients with histologically confirmed FIGO stage III or IV measurable epithelial ovarian carcinoma were enrolled in this study. This was a moderately to heavily pretreated patient population, which had had one (15 patients) or two (14 patients) prior chemotherapy regimens for advanced disease. All had received prior cisplatin and/or carboplatin therapy, and $84 \%$ were considered platinum-resistant because they had either failed to respond or had relapsed or progressed within 6 months of completing prior platinum-based therapy (Table 1). Disease progression or recurrence was documented by laparotomy, paracentesis, CT scan, or physical examination

Table 1. Patient characteristics

\begin{tabular}{lcc}
\hline Characteristic & $n=31$ & $(\%)$ \\
\hline Age, years & 59 & \\
Median & $39-74$ & \\
Range & & \\
Race, $n(\%)$ & 20 & $(65)$ \\
White & 6 & $(19)$ \\
Black & 4 & $(13)$ \\
Hispanic & 1 & $(3)$ \\
Asian & & \\
Performance status, $n(\%)$ & 9 & $(29)$ \\
0 & 20 & $(65)$ \\
1 & 1 & $(3)$ \\
2 & 1 & $(3)$ \\
Unknown & 1 & $(3)$ \\
Number of prior chemotherapy regimens, $n(\%)$ & $(45)$ \\
3 & 14 & $(38)$ \\
2 & 15 & \\
1 & 1 & $(84)$ \\
Adjuvant only & & \\
Disease status, $n(\%)$ & 26 & \\
Platinum-resistant & 5 & \\
Recurrent & & \\
\hline
\end{tabular}

*Recurred more than 6 months after completing prior platinum therapy. within one month prior to treatment with CI-973. Patients had not received chemotherapy or radiation therapy for at least 4 weeks prior to treatment. Patients who had received prior intraperitoneal chemotherapy or abdominal or pelvic radiation were not eligible for treatment with $\mathrm{CI}-973$. At baseline, patients had a creatinine clearance $>1 \mathrm{~mL} \mathrm{sec}^{-1}$, an ALT level $<$ twice the upper limit of normal of the institution, and a total bilirubin level $<34.2 \mu \mathrm{mol} \mathrm{L}{ }^{-1}$. Baseline hematologic criteria included an absolute granulocyte count of $>1.5 \times 10^{9} \mathrm{~L}^{-1}$ and platelet count of $>100 \times 10^{9} \mathrm{~L}^{-1}$. Measurements of hematologic parameters and blood chemistries were performed weekly during treatment. Urinalysis, measurement of serum CA125 levels, and creatinine clearance estimates were performed every 3 weeks. Monitoring for CI-973-related toxicity occurred throughout the study and an evaluation of toxicity occurred at the end of each treatment period. Toxicities were graded according to the National Cancer Institute common toxicity criteria.

\section{Drug administration}

CI-973 was administered as a 30-minute intravenous infusion once every 21 days. The starting dose was $190 \mathrm{mg} \mathrm{m}^{-2}$, which could be increased or decreased by $25 \%$ for subsequent doses, depending on each patient's tolerance of therapy. Patients who had progression were withdrawn from the study. Patients whose best response to therapy was stable disease could receive up to four courses of CI-973. Patients who achieved a complete or partial response to treatment received CI-973 until relapse or recurrence.

\section{Response criteria}

Sentinel lesions were measured either by physical exam or CT scan at baseline and 3 weeks after each dose. Objective response was determined by comparing the size of measurable sentinel lesions after at least one course of treatment to the size at baseline according to standard criteria for the evaluation of solid tumors ${ }^{(10,11)}$. A complete response (CR) was defined as the disappearance of all known disease. A partial response (PR) was a $50 \%$ or greater decrease in the sum of the product of cross-sectional diameters (tumor size) of sentinel lesions and no appearance of new lesions. Both complete and partial responses had to be confirmed at least 3 weeks after the initial assessment of response. Stable disease was defined as a less than $50 \%$ decrease and less than $25 \%$ increase in tumor size, with no appearance of new lesions. Progressive disease was defined as a $50 \%$ or greater 
increase in the size of any one sentinel lesion, or the appearance of a new lesion. Changes in CA125 levels were not included in definitions of response.

\section{Results}

Three of 31 patients $(9.7 \%)$ responded to treatment (Table 2), One responder had platinum-resistant disease. The patient was a 46-year old woman who had previously achieved a partial response to treatment with cisplatin plus cyclophosphamide. She achieved a CR on Day 67 after three courses of CI-973. She relapsed approximately 2 months later on Day 130 after having received a total of six courses of therapy. Her CA125 level remained elevated throughout treatment with CI-973. Two responders had recurrent ovarian cancer that was not proven to be platinumresistant. One such responder was a 44-year-old woman whose ovarian cancer had recurred approximately 4 years after she had received a regimen containing cisplatin, doxorubicin, and cyclophosphamide. The patient achieved a CR on Day 88 after four courses of CI-973 and her CA125 level normalized. She remained in CR through her sixth course of therapy (Day 170), then was lost to follow-up. The patient later developed recurrent disease and reinitiated therapy with CI-973 on Day 585 . The date of recurrence and thus the duration of response were unknown. She did not respond to four additional courses of therapy and was withdrawn from the study on Day 742 because of progressive disease. The patient died of her disease on Day 881, approximately 2.5 years after her first dose of CI-973. The second responder with recurrent disease was a 67-year old woman who had previously achieved a complete response to carboplatin plus cyclophosphamide. The patient achieved a CR on Day 56 after two courses of CI-973 treatment and her CA125 level had normalized by Day 77 . She received a total of 18 courses of CI-973 and has remained in CR for over one year (13.7 months).

No treatment-related deaths occurred. At last contact, 13 patients had died of their ovarian cancer. The median Kaplan-Meier survival time was estimated to be 224 days, or 7.4 months (Fig. 1).

Table 2. Rates of response to CI-973 therapy

\begin{tabular}{lcc}
\hline & $n$ & $(\%)$ \\
\hline Complete response* & 3 & $(9.7)$ \\
Partial response & 0 & \\
Stable disease & 17 & $(54.8)$ \\
Progressive disease & 11 & $(35.5)$ \\
\hline
\end{tabular}

*By measurable disease criteria. Normal CA125 levels were not required.

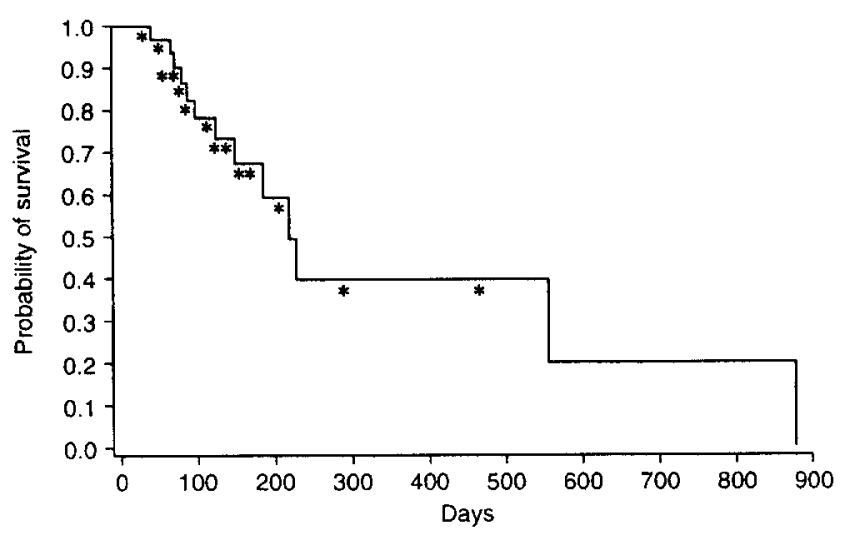

Fig. 1. Kaplan-Meier survival curve; *, a censored patient.

No patient was withdrawn from the study because of treatment-related toxicity. Neutropenia was doselimiting. The median nadir absolute neutrophil count was $1.200 \times 10^{9} \mathrm{~L}^{-1}$ and the nadir occurred a median of 15 days after dosing. Filgrastim was used at the investigators' discretion. Fifteen patients $(48 \%)$ experienced grade IV neutropenia $\left(<0.500 \times 10^{9} \mathrm{~L}^{-1}\right)$. The median time to recovery $\left(\geqslant 1.000 \times 10^{9} \mathrm{~L}^{-1}\right)$ was 7 days, with a range of $2-15$ days. Two patients experienced treatment-associated febrile neutropenia, and one of these patients subsequently developed sepsis. Both patients recovered within 5 days. Three other grade IV adverse events occurred in one patient each (vomiting, headache, and anemia). These events were not considered treatment-related.

The most frequently occurring CI-973-associated toxicities were neutropenia, nausea and/or vomiting, asthenia, anemia, abdominal pain, and diarrhea (Table 3 ). Most of the occurrences were mild or moderate. Neurotoxicity was infrequent and consisted largely of mild, reversible paresthesias. One patient experienced intermittent tinnitus, which was considered possibly treatment-related even though it was present at baseline. There were no cases of nephrotoxicity.

\section{Discussion}

CI-973 was developed with the goal of obtaining an anticancer agent that was active against cisplatin-

Table 3. Toxicities occurring in at least $20 \%$ of patients

\begin{tabular}{lll}
\hline Toxicity & $n$ & $(\%)$ \\
\hline Leukopenia & 26 & $(84)$ \\
Nausea and/or vomiting & 25 & $(81)$ \\
Asthenia & 12 & $(39)$ \\
Anemia & 9 & $(29)$ \\
Abdominal pain & 8 & $(26)$ \\
Diarrhea & 8 & $(26)$ \\
\hline
\end{tabular}


resistant tumors and was not associated with the doselimiting toxicities characteristic of cisplatin. Preclinical studies were promising: not only was the antitumor activity of CI-973 comparable to cisplatin and superior to carboplatin against cisplatin-sensitive tumor cell lines, CI-973 also exhibited a high degree of activity against several cell lines that were highly cisplatinresistant ${ }^{(4-6)}$. Animal toxicology studies showed that dose-limiting toxicity primarily targeted the hematopoietic system and gastrointestinal tract. Unlike cisplatin, CI-973 was not ototoxic and did not produce renal toxicity. There was also no evidence of neurotoxicity. Phase I studies in patients with solid tumors established that neutropenia was the doselimiting toxicity, and confirmed that Cl-973 was not seriously nephrotoxic, neurotoxic, or ototoxic ${ }^{(7-9)}$. Furthermore, it did not cause severe thrombocytopenia, even in patients who developed Grade IV neutropenia. The results obtained in this Phase 2 study have confirmed all of these previously reported data.

The Phase 2 program was designed to determine the level of antitumor activity of CI-973 against a variety of solid tumors. Ovarian cancer was chosen as one of the tumor types for study because of its relative sensitivity to initial treatment with platinum compounds and the problem of the development of platinum resistance ${ }^{(3)}$. Given that $\mathrm{CI}-973$ had in vitro activity against cisplatin-resistant cell lines, it was a promising candidate for the treatment of advanced ovarian cancer. However, the activity of CI-973 against advanced epithelial ovarian cancer in this study was minimal: the observed response rate was only $10 \%$ (3/ 31 patients) and in one patient the response was only of 2 months duration. Furthermore, two of the responses occurred among the group of five patients whose cancer was potentially platinum-sensitive. A response rate $\geqslant 20 \%$ would have justified further study of Cl-973 in ovarian cancer. Although the observed response rate of $10 \%$ was not statistically different from a response rate of $20 \%$ ( $Z$ value $=1.17$ ), the sample size of 31 patients provided only $47 \%$ power to detect a difference of $10 \%$. Therefore, these results do not support the development of CI-973 as single-agent therapy for this indication. In addition, they suggest that preclinical models of platinum resistance may not always correlate with clinical activity in patients with ovarian cancer. It may be necessary to better define such models to enhance the prospect of identifying a platinum analog with clinical activity in patients with refractory ovarian cancer. However, with its relatively mild toxicity profile, CI973 may be a suitable candidate for inclusion in a dose intensification chemotherapy regimen. For example, the dose-limiting neutropenia was of brief duration and was rarely associated with infection. Therefore, concurrent therapy with GCSF or GMCSF could be expected to overcome this toxicity and allow the use of higher, possibly more effective doses.

\section{References}

1 Omura GA, Brady MF, Homesley HD, et al. Long-term follow-up and prognostic factor analysis in advanced ovarian carcinoma: The gynecologic oncology group experience. J Clin Oncol 1991; 9: 1138-50.

2 Ozols RF, Rubin SC, Dembo AJ, Robboy S. Epithelial ovarian cancer. In: Hoskins WJ, Perez CA, Young RC, eds. Principles and Practice of Gynecologic Oncology. Philadelphia, JB Lippincott Co, 1992; 731-81.

3 Young RC, Fuks Z, Hoskins WJ. Cancer of the ovary. In: Devita VT, Hellman S, Rosenberg SA, eds. Cancer: Principles and Practice of Oncology, 3rd edn, Philadelphia, JB Lippincott Co, 1989; 1162-96.

4 Weiss $\mathrm{RB}$, Christian MC. New cisplatin analogues in development. Drugs 1993; 46: 360-77.

5 Kraker AJ, Moore CW, Roberts BJ, Leopold WR, Elliott WL. Preclinical antitumor activity of CI-973,[SP-4-3-(R)]-[1,1cyclobutanedicarboxylato(2-)](2 methyl-1,4-butane-diamineN, N')platinum. Invest New Drugs 1991; 9: 1-7.

6 Perez RP, O'Dwyer PJ, Handel LM, Ozols RF, Hamilton TC. Comparative toxicity of CI-973, cisplatin, carboplatin and tetraplatin in human ovarian carcinoma cell lines. Int J Cancer 1991; 48: 265-9.

7 Fukuoka M, Niitani H, Hasegawa K, et al. Phase I study of new platinum compound NK121. Proc Am Soc Clin Oncol 1989; 8: 62 .

8 O'Dwyer PJ, Hudes GR, Walczak J, et al. Phase I and pharmacokinetic study of the novel platinum analogue CI-973 on a 5-daily dose schedule. Cancer Res 1992; 52: 6746-53.

9 Theriault RL, Cohen IA, Esparza L, Kowal C, Raber MN Phase I clinical evaluation of [SP-4-3(R)]-[1.1-cyclo-butanedicarboxylato(2-] (2-methyl-1,4-butanediamine- $\mathrm{N}, \mathrm{N}^{1}$ ) platinum in patients with metastatic solid tumors. Cancer Chemother Pharmacol 1993; 31: 333-7

10 World Health Organization. WHO Handbook for Reporting Results of Cancer Treatment No. 48, Geneva: WHO offset publication 1979.

11 Miller AB, Hoogstraten B, Staquet M, Winkler A. Reporting results of cancer treatment. Cancer 1981; 47: 207-14.

Accepted for publication November 22, 1995. 